\title{
Electric dipole moment planning with a resurrected BNL Alternating Gradient Synchrotron electron analog ring
}

\author{
Richard M. Talman * \\ Laboratory of Elementary Particle Physics, Cornell University, Ithaca, New York 14853, USA \\ John D. Talman \\ UAL Consultants, Ithaca, New York 14850, USA \\ (Received 10 April 2015; published 22 July 2015)
}

\begin{abstract}
There has been much recent interest in directly measuring the electric dipole moments (EDM) of the proton and the electron, because of their possible importance in the present day observed matter/antimatter imbalance in the Universe. Such a measurement will require storing a polarized beam of "frozen spin" particles, $15 \mathrm{MeV}$ electrons or $230 \mathrm{MeV}$ protons, in an all-electric storage ring. Only one such relativistic electric accelerator has ever been built - the $10 \mathrm{MeV}$ "electron analog" ring at Brookhaven National Laboratory in 1954; it can also be referred to as the "AGS analog" ring to make clear it was a prototype for the Alternating Gradient Synchrotron (AGS) proton ring under construction at that time at BNL. (Its purpose was to investigate nonlinear resonances as well as passage through "transition" with the newly invented alternating gradient proton ring design.) By chance this electron ring, long since dismantled and its engineering drawings disappeared, would have been appropriate both for measuring the electron EDM and to serve as an inexpensive prototype for the arguably more promising, but 10 times more expensive, proton EDM measurement. Today it is cheaper yet to "resurrect" the electron analog ring by simulating its performance computationally. This is one purpose for the present paper. Most existing accelerator simulation codes cannot be used for this purpose because they implicitly assume magnetic bending. The new UAL/ETEAPOT code, described in detail in an accompanying paper, has been developed for modeling storage ring performance, including spin evolution, in electric rings. Illustrating its use, comparing its predictions with the old observations, and describing new expectations concerning spin evolution and code performance, are other goals of the paper. To set up some of these calculations has required a kind of "archeological physics" to reconstitute the detailed electron analog lattice design from a 1991 retrospective report by Plotkin as well as unpublished notes of Courant describing machine studies performed in 1954-1955. This paper describes the practical application of the ETEAPOT code and provides sample results, with emphasis on emulating lattice optics in the AGS analog ring for comparison with the historical machine studies and to predict the electron spin evolution they would have measured if they had polarized electrons and electron polarimetry. Of greater present day interest is the performance to be expected for a proton storage ring experiment. To exhibit the ETEAPOT code performance and confirm its symplecticity, results are also given for 30 million turn proton spin tracking in an all-electric lattice that would be appropriate for a present day measurement of the proton EDM. The accompanying paper "Symplectic orbit and spin tracking code for all-electric storage rings" documents in detail the theoretical formulation implemented in ETEAPOT, which is a new module in the Unified Accelerator Libraries (UAL) environment.
\end{abstract}

\section{INTRODUCTION}

Motivation for electric storage ring "traps" for electrons or protons. - The U.S. particle physics community has recently updated its vision of the future and strategy for the next decade in a Particle Physics Project Prioritization Panel (P5) Report. One of the physics goals endorsed by P5

\footnotetext{
*richard.talman@cornell.edu

Published by the American Physical Society under the terms of the Creative Commons Attribution 3.0 License. Further distribution of this work must maintain attribution to the author(s) and the published article's title, journal citation, and DOI.
}

is measuring the EDM of fundamental particles (in particular, accepting them as "fundamental," proton, deuteron, neutron and electron).

Since Standard Model EDM predictions are much smaller than current experimental sensitivities, detection of any particle's nonzero EDM would signal discovery of new physics. If of sufficient strength, such a source could support an explanation for the observed matter/antimatter asymmetry of our Universe. A proton EDM collaboration [1] has proposed a storage ring proton EDM measurement at the unprecedented level of $10^{-29} e-\mathrm{cm}$, an advance by nearly 5 orders of magnitude beyond the current indirect bound obtained using $\mathrm{Hg}$ atoms. 
The proposed EDM measurement is based on the accumulation of tiny "wrong-plane" (i.e., "right" for EDM, "wrong" for MDM) spin precessions that can be accounted for only by a nonzero EDM. Polarized beams can be frozen (for example in longitudinally polarized states) in storage rings containing appropriate combinations of electric and magnetic bending elements; the relative amount depends on the magnetic dipole moment of the particles being stored. Only for a few particles, of which the electron and the proton are the most important, can the spins be frozen in purely electric rings. This is highly advantageous, since such rings support beams circulating both clockwise and counterclockwise, permitting measurements for which important systematic errors cancel. The electric dipole moment (EDM) of the proton is known to be so small that, in order to reduce systematic errors enough to measure it in a storage ring requires measuring differences between counter-circulating beams. This can be done sequentially or, to provide differential beam position precision at the cost of beam-beam complications, with simultaneous circulating beams. (Direct colliding beambeam effects are estimated to be negligible [1].)

A comparably important advantage of electric bending is that the absence of intentional magnetic fields will reduce the presence of unintentional (radial) magnetic field components, which are expected to be the dominant source of spurious precession, mimicking the EDM effect.

To freeze the spin procession, conventional storage ring bending magnets are replaced with the corresponding electric elements. Though the circulating particles are constantly being attracted to the inner electrode, their centrifugal force causes them to circulate indefinitely in a more or less circular orbit. This establishes the storage ring as an electric "trap," albeit for an intense moving bunch rather than for a few slow particles.

In a storage ring EDM measurement, bunches of longitudinally polarized protons will circulate for "long" time intervals such as $1000 \mathrm{~s}$. Because of inevitable parameter spreads, individual particle spins will precess differently and, after a spin coherence time (SCT), the beam polarization will have been attenuated (due to decoherence) to a point where the EDM precession rate has become unmeasurably small. The wrong-plane polarization difference between early and late times, when ascribed to the torque of the bend field acting on the electric dipole moment, will provide a measurement of the proton EDM.

For measuring EDM's the accumulation and measurement of small effects requires analysis, mitigation and control of various systematic errors. Issues to be studied with ETEAPOT include: (i) radial B-field: since the torque due to any residual radial B-field acting on the magnetic dipole moment (MDM) mimics the EDM effect, this is expected to be the dominant systematic error; (ii) geometric phase: some EDM-mimicking precessions would average to zero except for the noncommutativity of $3 \mathrm{D}$ rotations; (iii) nonradial E-field, vertical quad misalignment, rf cavity misalignment etc.: these cause the closed orbit to deviate from design. The systematic errors they cause cancel to "lowest order" but higher order effects need to be investigated. (iv) Polarimetry: realistic beam distributions need to be used to identify and reduce left-right asymmetry bias, which is another source of systematic error.

Initial storage ring simulation tasks.-Initial tasks for the ETEAPOT code are to simulate the performance of arbitrary electric storage rings. As with any storage ring, these tasks (which now include also spin tracking) are: (1) evaluation of linearized lattice functions such as tunes, the Twiss, $\alpha, \beta$, and $\gamma$ functions, dispersion function, and spin tunes, as well as chromatic dependence of these functions, and their sensitivity to imperfections; (2) short term tracking, for confirmation of the lattice functions, for determining dynamic aperture, and for investigating the performance of analytically derived spin decoherence compensation schemes; (3) guaranteed to be stable, long term tracking. The EDM experiment requires SCT to be $1000 \mathrm{~s}$ or longer. During this time every particle executes about $10^{9}$ betatron oscillations. The code is required to exhibit negligible spurious growth or decay for this interval of time.

Compared to magnetic bending, electric bending complicates these tasks. This requires the ETEAPOT treatment to differ from the well-established TEAPOT treatment. As implemented in UAL/TEAPOT [2], lattice function determinations use a truncated power series formalism that has not (as yet) been established for electric rings. Translating the arbitrary order formalism from magnetic to electric elements is a huge task that has only just begun. To cover all three storage ring simulation tasks on the time scale required for EDM planning we have proceeded as follows.

We implement exact (and therefore exactly symplectic) tracking in the inverse square law electrical field between spherical electrodes. The optimal field shape is currently unknown, however, and there is no reason to suppose that inverse square law dependence is optimal; the actual electric field shape will presumably be different. This is handled by introducing artificial virtual quadrupoles in the interiors of bend elements, in order to model deviation of the actual electric field away from the idealized representation. Real quadrupoles are typically also present in the lattice, for example to alter the lattice focusing, or to control dispersion. Thin sextupoles (present for example to adjust chromaticities or to compensate spin decoherence effects) and other thin multipole elements will also be present. Causing only "kicks," these thin elements, either real or virtual, also preserve symplecticity. Now complete, this track allows tasks (2) and (3) to be completed for arbitrary lattices.

Since (linearized) transfer matrices are not introduced to describe particle evolution, the ETEAPOT code cannot be used to extract Twiss functions directly, as is commonly done in conventional Courant-Snyder accelerator formalism. However, by tracking a small set (actually 21 particles) tunes and Twiss functions can be obtained (to amply satisfactory accuracy) using postprocessing with FFT and MIA (model-independent analysis). [There are (appropriately small) user-defined parameters in the ETEAPOT 
code-xtyp, xptyp, ytyp, yptyp, cttyp, detypthat establish scales for this postprocessing.] Now complete this code also provides transfer matrix determination which, in turn, allows determination of all the lattice functions required to complete task (1).

After comparing ETEAPOT simulation results to results measured on the Alternating Gradient Synchrotron (AGS) analog ring this paper makes a start on the sort of beam evolution studies that will be needed for analyzing storage rings capable of measuring electrical dipole moments. Accurate spin evolution (as predicted by the BMT equation) is obviously essential. In the ETEAPOT approach the orbit is constructed by smoothly joining arc segments. Each of these segments lies in a single plane; this permits the BMT equation to be solved analytically in closed form. With this the spin evolution is also subsumed into the smooth joining of arc segments.

There will be two main applications for simulation codes like ETEAPOT. One will be to aid in the design of compensation schemes to increase SCT, as will be required to improve the accuracy of EDM measurements. The other will be to simulate the spin phase locking schemes that will also be essential. The storage ring will, after all, be a trap that needs to be understood with the same level of sophistication as the tabletop traps that have been so important in precision determination of elementary particle parameters. Studies like this have not yet been started.

\section{THE AGS ELECTRON ANALOG RING}

Of the more than 100 relativistic accelerators ever built, only one has used electric rather than magnetic bending. It was the AGS electron analog machine at BNL. Curiously it was also the first ring ever to use alternating gradient (AG) focusing. (The Cornell $1.1 \mathrm{GeV}$ alternating gradient electron ring was commissioned at more or less the same time and the BNL AGS ring itself somewhat later.)

Along with using electrons instead of protons, and limited by achievably high electric field, cost minimization of the AGS analog led to the choice of $10 \mathrm{MeV}$ maximum

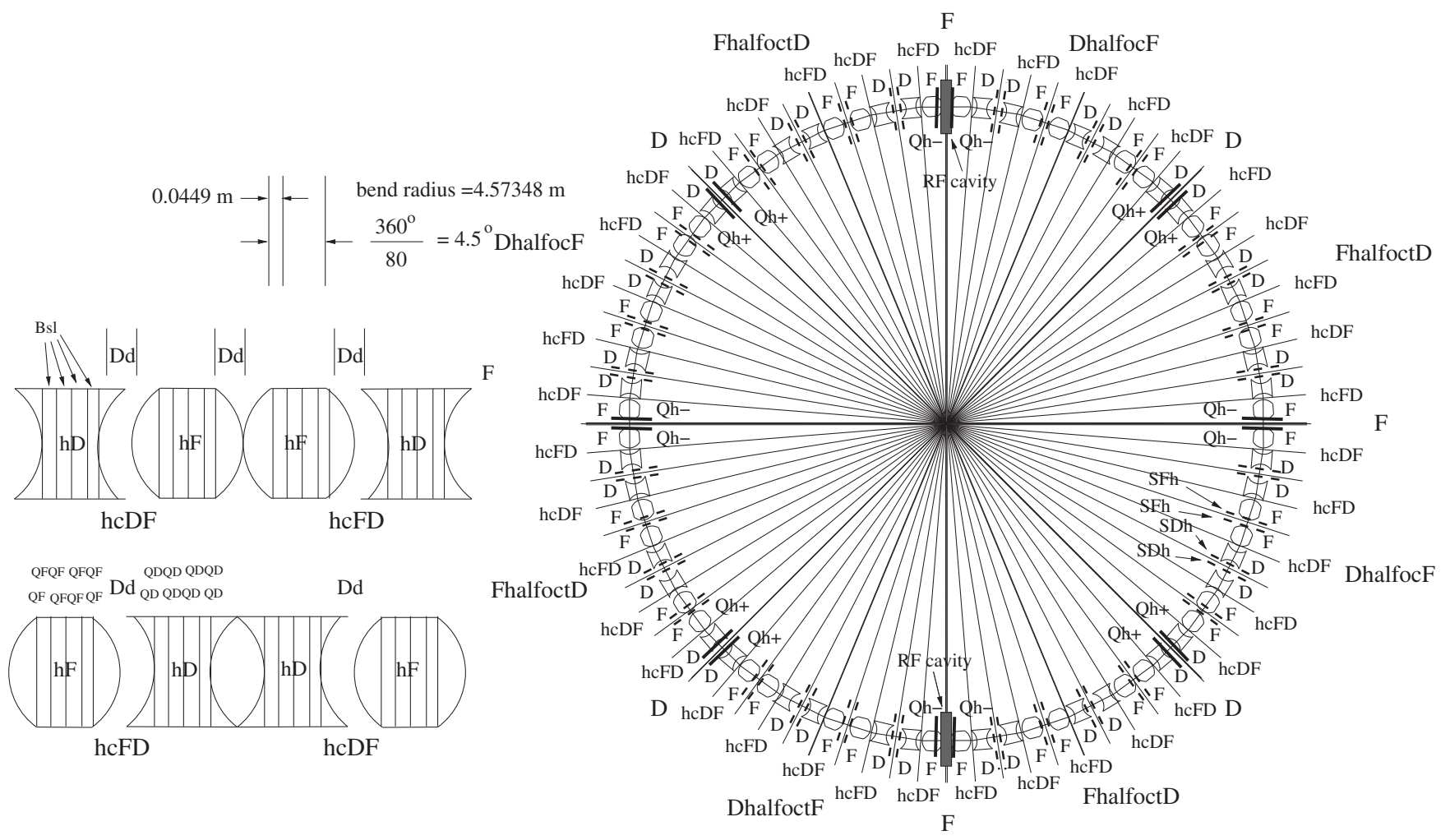

FIG. 1. The 1955 AGS analog lattice as reverse engineered from available documentation-mainly the 1953 proposal letter from BNL Director Haworth to AEC Director of Research Johnson. Individual "lenses" (as they were referred to in the original documentation; in modern terminology they would be referred to as "combined function bends") are shown on the left; vertical lines indicate locations of thin quadrupoles present in the lattice description to represent the focusing effect of the electric lens elements. Elements are identified by ad hoc labels assigned in the reconstruction. They are not obtained from the original lattice design, but are needed for modern day lattice description files. Lattice description files are available (for example from the authors) in various formats: E_AGS_Analogue2.xm 1 gives the pristine design with all parameters in algebraic (or function of algebraic) form. E_AGS_Analogue2. adxf is the same, but with all parameter values, though ideal, converted to numerical values. E_AGS_Analogue $\overline{2}$. sxf contains the fully instantiated lattice description (with parameters from otherwise identical elements allowed to be individualized). Most UAL lattice files are available in all these forms. 
electron energy, $4.7 \mathrm{~m}$ bending radius, $6.8 \mathrm{MHz} \mathrm{rf}$ frequency, and $600 \mathrm{~V}$ rf voltage. These optimization considerations are very much the same as will be used to fix the parameters of a frozen spin proton ring (which is tentatively expected to have a bend radius of about $50 \mathrm{~m}$ ) [1]. The UAL/ETEAPOT code (documented in the accompanying paper) was developed with this application in mind. The present paper gives initial results.

Starting from fragmentary documentation, especially the original proposal letter from the BNL director to the AEC, which is attached as an Appendix, this research started by reverse engineering the AGS analog lattice and producing a lattice description file E_AGS_Analogue.sxf, in the format needed for processing in the Unified Accelerator Libraries (UAL) environment. Results obtained using ETEAPOT are then compared with measurements performed on the ring at BNL in 1954-1955.

By chance, the magic kinetic energy for freezing electrons, which is $15 \mathrm{Mev}$, is not very different from the $10 \mathrm{MeV}$ of the AGS analog ring. So that ring could have been used to measure the electron EDM just by increasing the electric bend field by a factor of 1.5. Morse [3] and others have suggested building such a ring for this purpose. The ETEAPOT code can therefore be used to simulate an electron EDM measurement using a ring whose successful performance as a storage ring is all but guaranteed by the successful operation of an "identical" ring in $1955.15 \mathrm{MeV}$ is a very convenient electron energy and a high quality electron source would be available, for example as described by Bazarov [4].

The present paper describes our "resurrection" of AGS analog ring from historic BNL documentation, and simulates its performance with codes intended for the proton EDM experiment.

A quite superficial (day long) search of the BNL library and the Accelerator-Collider report library found one quite extensive report, produced retrospectively in 1991 by Martin Plotkin [5], and a few ancient reports describing machine studies results. Also a report privately communicated from Ernest Courant [6] contains experimental data to be simulated in this paper.

Before starting on this project, one of the authors, RT, benefited from three brief but valuable meetings with Ernest Courant, perhaps the father, or at least one of the parents, of the electron analog ring, inquiring about his recollections concerning the dynamics and performance of the ring. This contributed to our reconstruction. Bill Morse [7] reports having had a similar conversation with Courant a few years earlier. Bill recalls asking Ernest whether, back in 1953, he (Courant) understood the difference between electric and magnetic focusing. Ernest replied "of course" in his always kindly, but in this case somewhat exasperated, tone of voice.

Historical BNL documents. - The "Conceptual Design Report" for the AGS analog electron ring was a four page letter, dated August 21, 1953, from BNL Director Haworth

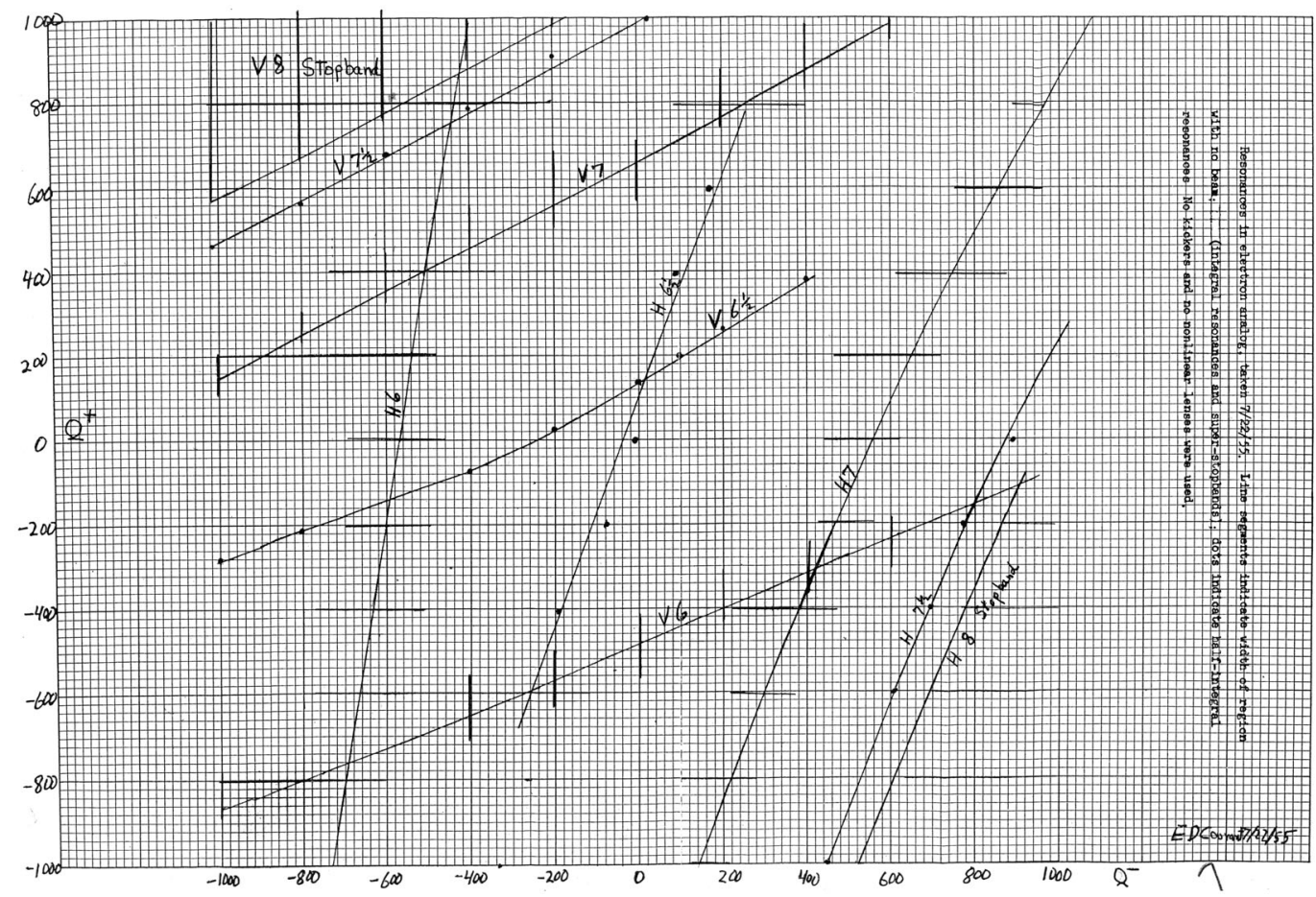

FIG. 2. Tune plane resonance diagram measured during machine studies at the AGS analog ring and reported by Courant [6]. A more polished and complete version of this plot, copied from Plotkin [5], is shown in Fig. 13 but with minor relabelings. This was published with permission of Brookhaven National Laboratory. 


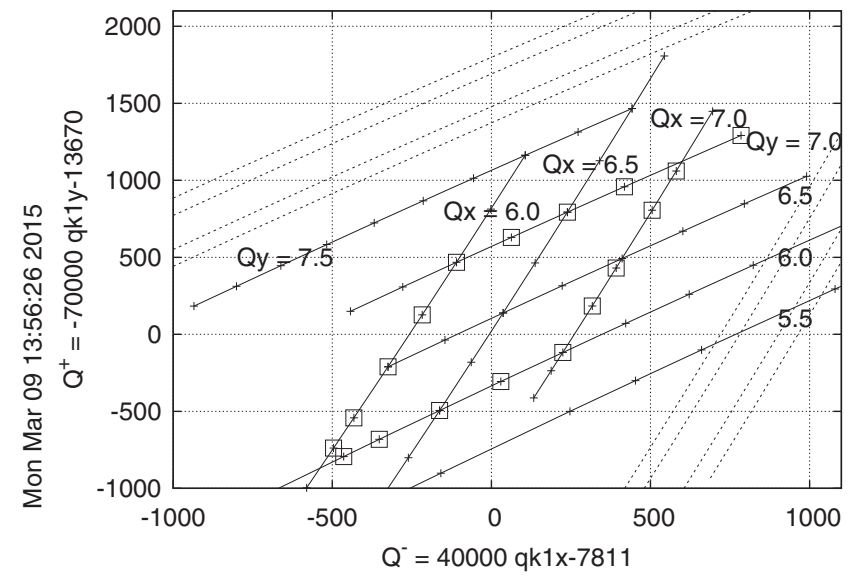

FIG. 3. Tune plane resonance diagram as calculated by TEAPOT, with bend elements treated as magnetic. There is good qualitative and quantitative agreement with the Courant data shown in Fig. 2.

to the A.E.C. (predecessor of D.O.E.) Director of Research Johnson, applying for funding. The letter is reproduced in its entirety in Fig. 8 in the Appendix. As brief as it is, this letter along with hints from Plotkin and Courant, includes everything needed to reconstruct the ring, as shown in Fig. 1. By 1955 the ring had been approved, built, and commissioned, and had achieved its intended purpose.

Following the Haworth letter in the Appendix are two other especially informative figures from the paper by Plotkin. Figure 12 is especially useful for visualizing the physical layout of the AGS Analogue ring and its vacuum system. Figure 13 is a more polished version of Fig. 3 which plots tune scans actually performed on the AGSAnalogue ring and reported by Ernest Courant in a July 28, 1955 BNL technical report [6].

In these plots, points of observed beam loss and observed beam disruption in the $\left(Q_{x}, Q_{y}\right)$ tune plane are correlated with expected resonances. Beam loss occurs on integer resonances, beam disruption occurs on half integer resonances.
The Courant report on, and analysis of, data collected in machine studies less than two years after the submission of the ring funding proposal, would certainly deserve an $\mathrm{A}+$ grade by modern machine studies grading standards.

The axes of Figs. 2 and 3 are voltages (proportional to quadrupole family focusing strengths) applied to the tuneadjusting quadrupole families. Short heavy lines indicate regions with no beam survival (presumably due to integer resonance). Dots indicate points reported by Courant as "showing the characteristic double envelope of the oscilloscope pattern, sometimes accompanied by beam loss." (These were presumably due to "very narrow" $1 / 2$ integer resonance.) The nominal central tunes values are $\left(Q_{x}, Q_{y}\right)=(6.5,6.5)$. Stop bands due to the eightfold lattice symmetry are also shown.

\section{CURRENT DAY SIMULATION OF 1955 MACHINE STUDIES TUNE PLANE SCAN}

Our AGS analog lattice reconstruction is shown in Fig. 1. The Courant tune plane plot is shown in Fig. 2. It is to be compared with a similar plot, simulated by TEAPOT and shown in Fig. 3. As far as we know this code and this lattice representation are completely equivalent to Courant's model and analysis in 1955.

In the TEAPOT tune plane plot, boxes indicate points on integer resonance boundary curves of the stable diamond centered on nominal tune values $\left(Q_{x}, Q_{y}\right)=(6.5,6.5)$. Points lying on $1 / 2$ integer resonance lines are indicated by dots. Superperiodicity (eightfold periodicity) causes resonances with $Q_{x}=8$ or $Q_{y}=8$ indicated by broad blank bends bounded by narrowly spaced lines. Courant refers to these as "stop bands." Horizontal/vertical axes $\left(Q^{+}, Q^{-}\right)$are "electrode voltages on the quadrupoles in odd/even-numbered tanks." (For the meaning of "tank" see Fig. 12.) The quadrupole strength coefficients for variable quadrupoles $\left(Q^{+}, Q^{-}\right)$were determined empirically to match the central tunes. This means that absolute focusing
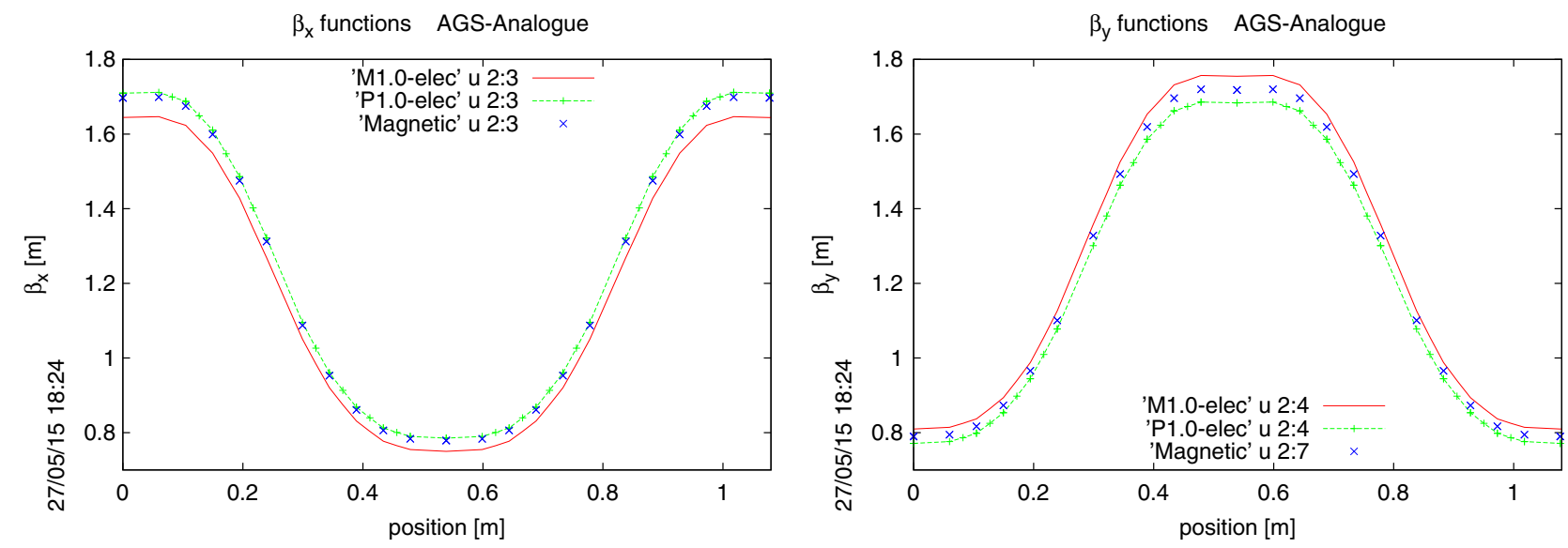

FIG. 4. Comparison for AGS Analogue2. sxf lattice with bends treated as electric $m=1$ (indicated by $\mathrm{P} 1.0$ ) or $m=-1$ (indicated by M1 . 0) or magnetic. $\beta_{x}$ is plotted on the left, $\beta_{y}$ on the right. As explained in the text, because of the strong focusing in the AGS analog lattice, switching from magnetic to electric bending causes only minor changes to the lattice functions. 
strength scales are not checked. Otherwise there are no significantly adjustable empirical lattice parameters.

The AGS analog ring provides only a coarse test of ETEAPOT since, for strong focusing lattices, the change from electric and magnetic bending is quite minor. This is illustrated in Fig. 4 which shows that the $\left(Q_{x}, Q_{y}\right)=$ $(6.5,6.5)$ tunes in the AGS analog ring are high enough that the tune plane structure is quite insensitive to whether the bends are treated as magnetic or electric.

Treating the difference between electric and magnetic focusing perturbatively, the implied tune difference depends inversely on the ring beta function (which itself varies inversely with tune, which is 6.5 in this case) at the location of the difference. For the eventual proton EDM ring the vertical tune has to be reduced from $Q_{y}=6.5$ by at least a factor of 10 . This amplifies the difference between electric and magnetic focusing, invalidating any such perturbative estimation and requiring sensitive readjustment of quadrupole strengths. Reducing $Q_{y}$ from 6.5 to 2.25 has been straightforward but, as the electric focusing became increasingly important, to decrease $Q_{y}$ further will require substantial design effort.
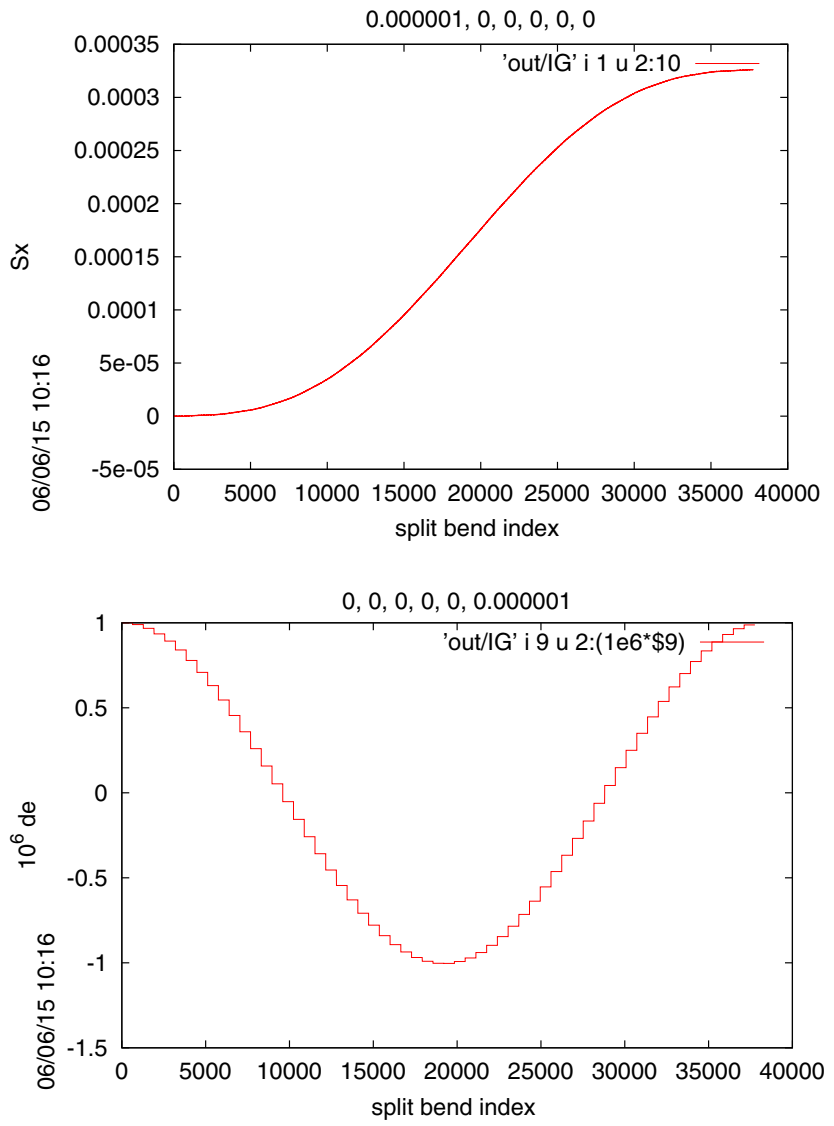

Spin evolution in the AGS analog ring is shown in Fig. 5 and described in the caption. These results begin to give us confidence in our understanding of electric rings and of our ability to simulate their performance using ETEAPOT. Substantial benchmarking and comparison with other tracking codes needs to be done to provide further confidence. For example it will be important to compare results with Runge-Kutta codes which, though inappropriate for long term tracking, can be very accurate and reliable for short term tracking.

\section{LONG TERM TRACKING IN A PROTON EDM STORAGE RING TRAP}

Plots so far have simulated the evolution of $10 \mathrm{MeV}$ electrons in the AGS analog electron ring. These roughly indicate the performance to be expected in a modern day electron EDM storage ring measurement. Of more immediate interest is the expected behavior of $230 \mathrm{MeV}$ protons in a significantly larger, but still all-electric proton EDM storage ring.
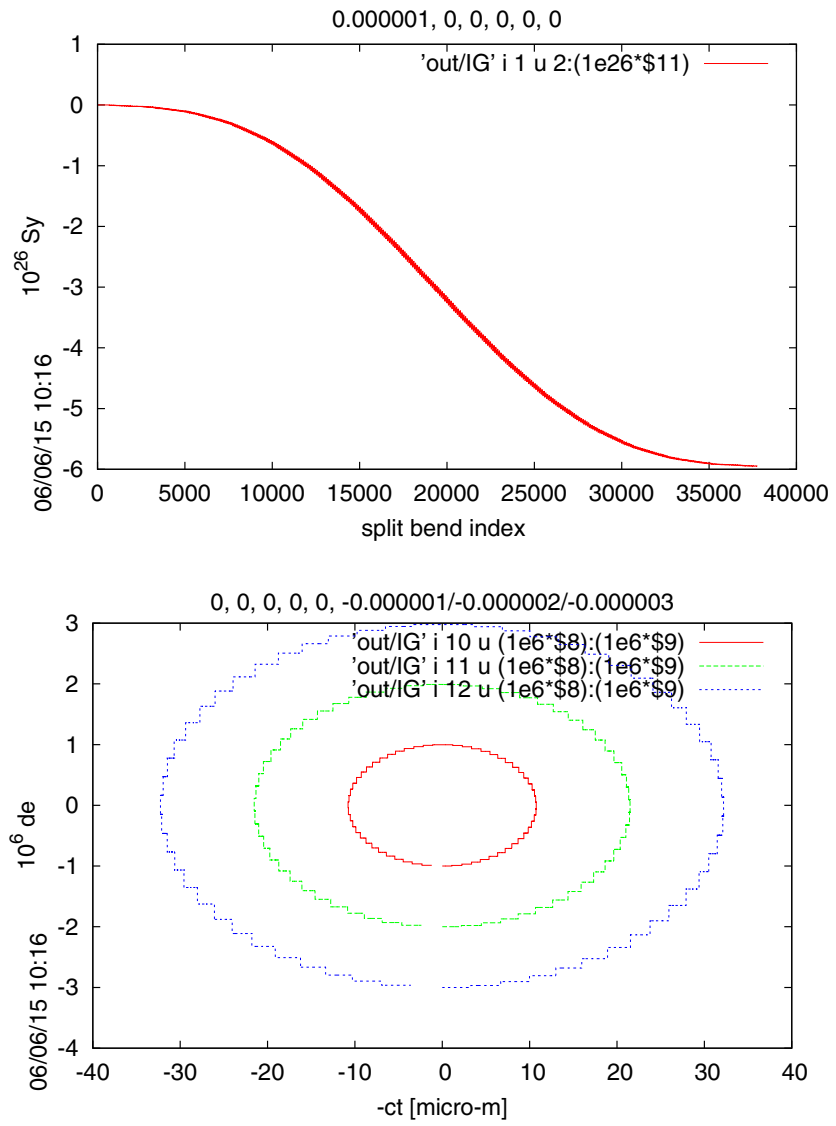

FIG. 5. The upper plots show the $s_{x}$ and $s_{y}$ spin components of a single electron, with initial horizontal amplitude xtyp $=1 \mathrm{e}-6 \mathrm{~m}$, in the AGS analog ring. (The 6D initial conditions are given above the plots.) The horizontal axis "split bend index" increases by 1 at each bend, of which there are 640, so about 59 turns are shown. The lower right figure shows longitudinal phase space evolution for three offenergy particles, with energy offset de plotted against time offset $-c t$. One sees that almost exactly one revolution is completed during this time. The synchrotron tune is therefore approximately $1 / 59$. The lower left plot shows the de energy deviation during one synchrotron oscillation period. 
Two considerations are especially important, both having to do with long time tracking. Simulating practical storage ring EDM measurements will require tracking times of about $1000 \mathrm{~s}$. Spurious damping or antidamping will limit the duration of reliable tracking. Almost as important will be tracking time limitation imposed by computation speed.

A single particle tracking example, taken from a recent proton EDM study, is illustrated in Fig. 7. The particle orbit and spin components are tracked around a prototype proton EDM storage ring for 33 million turns using ETEAPOT. (The number 33 million is arbitrary-it is when the run was stopped. But "bigness" of 33 s reflects the duration of a typical EDM run during which the spins need to be tracked and for which any spurious orbit damping or antidamping has to be acceptably small.)

The lattice being tracked is racetrack shaped, with long straight sections north and south for injection and various instrumentation. The circumference is $278 \mathrm{~m}$. It is shown in Fig. 6. By rolling the Möbius insert in the south straight section the ring can be converted from purely uncoupled to Möbius operation. (In Möbius operation vertical and horizontal betatron oscillations are interchanged every turn. This reduces the phase space dimensionality from 6 to 4 , simplifying compensation schemes for increasing the spin coherence time. More important, there is a substantial cancellation between horizontal-induced and verticalinduced spin decoherence.) The tracking was performed in Möbius mode. As stated earlier the tracking is exact and no artificial "symplectification" is applied. Any spurious damping or antidamping of the spatial orbit is less than ten percent over the 33 million turns. The (unit-magnitude) spin vector $\mathbf{S}$ is initially purely tangential (forward) and the vector magnitude never changes from its initial value of 1 .

It is characteristic of spin evolution for the transverse spin components to change over narrow ranges and over larger (but still small) ranges in synchronism with synchrotron oscillation. The upper left graphs of Fig. 7 show this, along with a sinusoidal fit with the parameters shown. These precessions are not expected to contribute significantly to spin decoherence. But any systematic growth over millions of turns will eventually lead to beam decoherence and limited spin coherence time.

An important computational task in planning to measure the proton EDM is to determine the spin coherence time SCT of the circulating beam. This is the time after which inevitable spreads in beam parameters will have attenuated the beam polarization significantly due to decoherence in the spin propagation. As long as both $S_{x}$ and $S_{y}$ magnitudes remain small, $\mathbf{S}$ remains in the forward hemisphere, and decoherence is suppressed.

One sees from the graphs of Fig. 7 that both $S_{x}$ and $S_{y}$ gradually deviate from zero. But, for the particular particle being tracked, these deviations are limited to small values. In particular, since $S_{x}^{2}+S_{y}^{2}$ remains always much less than 1 in magnitude, $S_{z}$ therefore remains not much less than 1 . So the beam polarization remains always in the forward hemisphere. Depolarization is therefore unimportant for particles of amplitude comparable to, or smaller than, this particle's. The tracking is therefore consistent with the SCT value exceeding the 33 million turns shown.

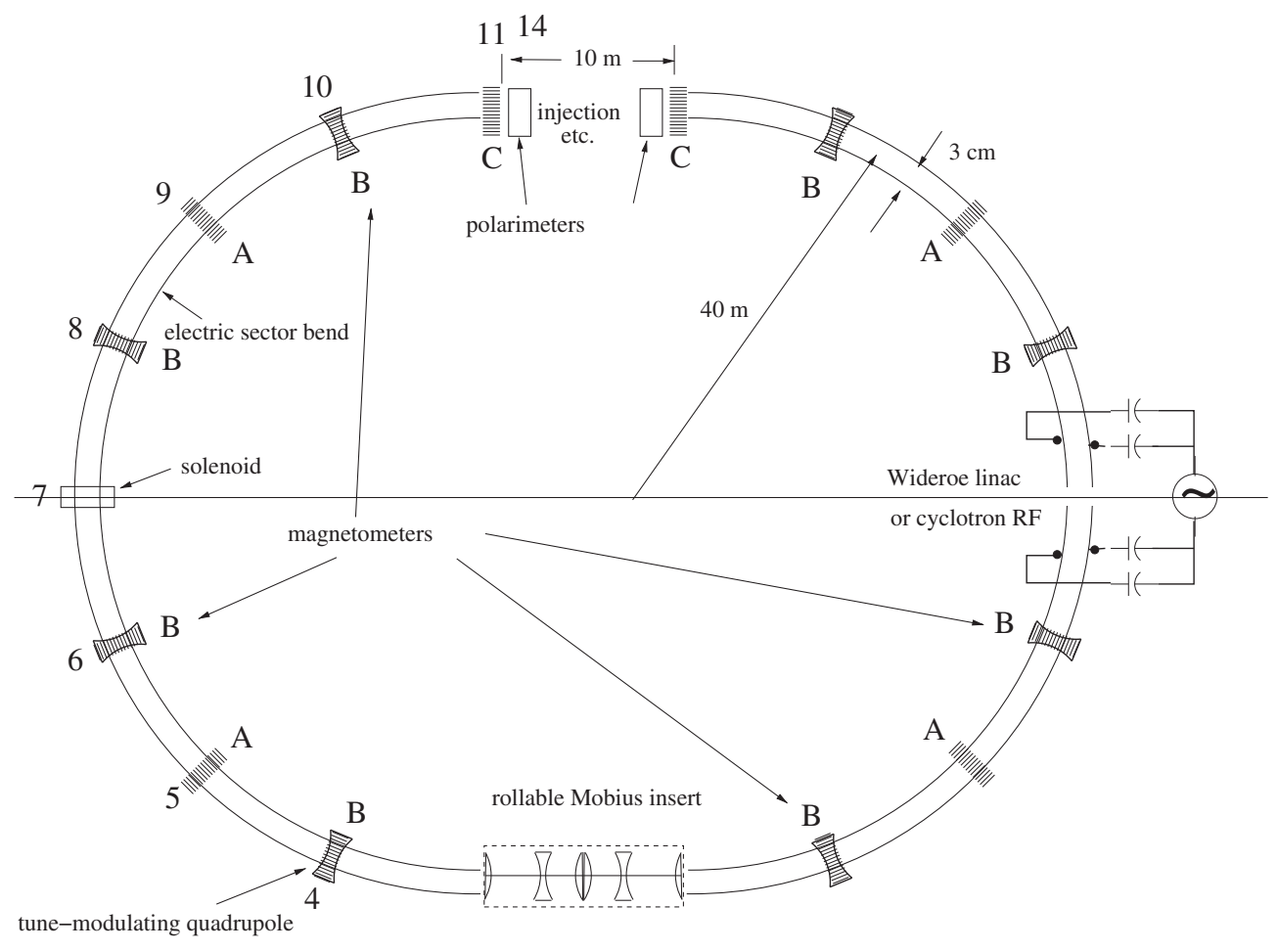

FIG. 6. Lattice for the long term tracking exhibited in Fig. 7. With the Möbius insert rolled by 45 degrees, horizontal and vertical betatron oscillations interchange every turn. 

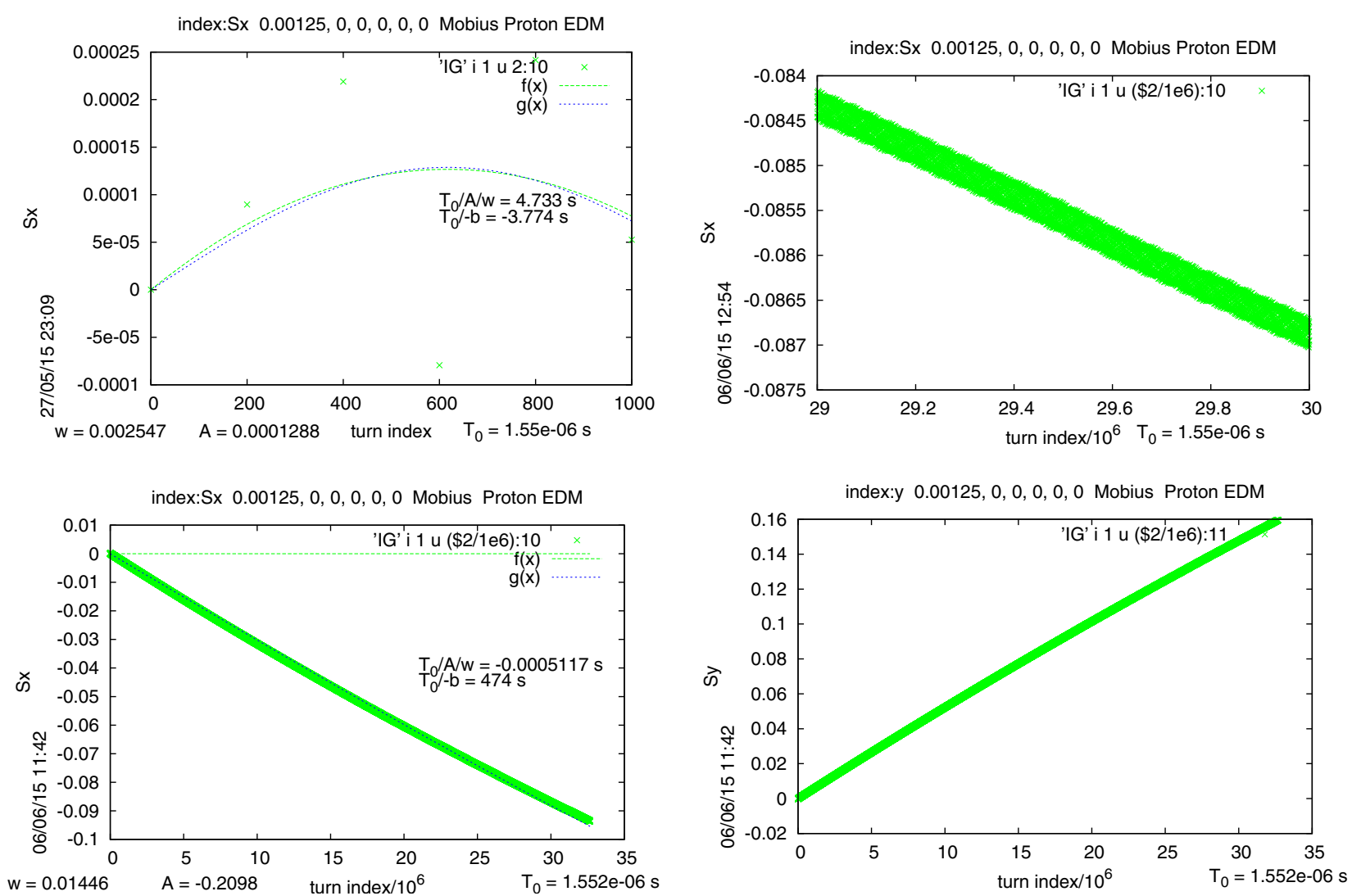

FIG. 7. Evolution of spin components for 33 million turns. Because $S_{z}=1$ initially, both $S_{x}$ and $S_{y}$ vanish at $x=0$, where $x$ is turn number. The upper left figure shows $S_{x}$ for the first 1000 turns, as fit by a sinusoid $A \sin (w x)$ where the horizontal axis variable $x$ is, in this case, turn number. The upper right figure shows $S_{x}$ for one million turns starting at $x=29$ million. The lower left and lower right figures show the evolution of $S_{x}$ and $S_{y}$ for the full 33 million turn run. Though these motions are oscillatory the oscillation amplitudes are less than the linewidth. The lattice file is E_pEDM-rtr1-Mobius.RF.sxf.

The lattice investigated in Fig. 7 is a "Möbius lattice" in which the horizontal and vertical betatron oscillations interchange on every turn. This strongly suppresses spin depolarization because precession in horizontal and vertical oscillation phases tend to cancel on a turn-by-turn basis. By fine-tuning this cancellation it is anticipated that extremely long SCT values can be obtained.

This will leave beam energy spread as the dominant source of beam depolarization. Fringe fields at bend elements are one such significant source of spin precession slewing. In this respect spin tracking is more sensitive than orbit tracking. Fringe fields are not very important in orbit calculations. Treating bends as "hard edged" mainly causes small tune shifts which are not very important, since both tunes are always set operationally using spectrum analysis. But, because of the spin precession sensitivity, in ETEAPOT, fringe fields are treated as linear ramps of length comparable with the gap between the electrodes, and not by hard edges. In any case synchrotron oscillation averaging also strongly suppresses this fringe field depolarization. This places further demands on longitudinal particle tracking, which is always delicate, because nonlinearity of synchrotron oscillations contributes to spin decoherence.

The particle revolution period is about one microsecond so the plots shown correspond to about thirty seconds of real clock time in the laboratory. On a laptop computer this computation takes a few hours per particle. The ratio of computation time to real laboratory time for a single particle is in the range from one to ten thousand. The present ETEAPOT single particle tracking approach is sufficient for early design tasks.

EDM storage ring beams will contain perhaps $10^{8}$ particles. Adequately precise simulation of the EDM experiment will require fewer than this, but at least thousands of particles to be tracked for significantly longer times than shown. This will require heavy parallelization of the tracking [8]. With little particle-to-particle interaction, the code is easily parallelizable. For more advanced tasks, such as investigating polarimeter biases or emittance growth caused intrabeam scattering, an efficient and scalable map-based computational approach is under consideration.

\section{SUMMARY}

As already mentioned in the abstract, this paper complements the preceding paper [9], which provides a technical explanation of the ETEAPOT code. This paper illustrates the practical use of that simulation code. ETEAPOT is a module in the Universal Accelerator 
Libraries (UAL) code environment [2]. It is the need for "freezing the spins" that makes it necessary to use allelectric storage rings, and it is electric bending that makes a code like ETEAPOT necessary.

We hope the historical material concerning the AGS analog ring will be of interest to members of the accelerator community. At a minimum, reviewing the successful experience with that ring should ease concerns about the practicality of building an all-electric storage ring nowadays.

But what has been described here is only a beginning, as regards the detailed operation of an electric ring, much less how the EDM can be measured once the storage ring has been commissioned. Much benchmarking and code comparison has to be completed concerning storage ring operation. Some that has already been accomplished is described in Ref. [10]. Once ring commissioning has been accomplished, the even greater task of reducing systematic errors in the EDM measurement will be left. Some planning for this is described in Ref. [8].

\section{ACKNOWLEDGMENTS}

This work has been partially supported by the U.S. DOE, OSP, Grant No. DE-SC0008891.

\section{APPENDIX}

Attached in the form of Figs. 8-11 is the Haworth letter mentioned in the text. Following that are two other especially informative figures from the report by Plotkin. Figure 12 is especially useful for visualizing the physical layout of the AGS Analogue ring and its vacuum system. Figure 13 is a more polished version of Fig. 3 which plots tune scans actually performed on the AGS-Analogue ring and reported by Ernest Courant in a July 28, 1955 BNL technical report [6].

BNL Director Haworth Electric Analogue Proposal Letter to AEC

August 21, 1953

\begin{abstract}
Dr. T.H. Johnson, Director
Division of Research

U.S. Atomic Energy Commission

Washington 25, D.C.

Dear Tom:

This lettex concerns certain aspects of our accelerator development program, particularly the proposed electron model.

As you know, the general development of a very high energy alternating gradient synchrotron is proceeding actively at Brookhaven, utilizing operating funds allocated to Basic Physics Research. As I explained in my letter of August 12, however, these funds are insufficient to carry forward the development as rapidly as desirable. Also, there are certain steps which should be taken for which the expenditure of operating funds is not appropriate. The first and most important of these is the construction of an electron model intended to provide final assurance of the technical feasibility of the chosen machine and, more importantly, to provide information enabling us to design in the most effective and economical manner. (We have no doubt of the general feasibility of accelerators of this type.)

We have given considerable thought to the requirements for such a model and to the philosophy which should guide us in designing and building it. In the alternating gradient synchrotron, two problems require especially careful exploration by extensive calculation and experimental modelling. These are the close-spaced resonances in the betatron oscillations and the shift of. phase stability at intermediate energies. It seems best to study these problems with an electron accelerator which would be essentially a: analogue rather than an exact model. This device should, in our opinion, be designed to yield the maximum of orbital data with a minimum of engineering complications, especially those not applicable to a final machine. After considerable thought we have arrived at a tentative description and list of parameters which follow.
\end{abstract}

The device would consist of an accelerator having an orbital radius of 15 feet and an overall diameter including the straight sections, of approximately 45 feet; the guide and focussing fields would be electrostatic, with electrode shapes as indicated in the sketch (full scale).

FIG. 8. This figure, continued in the following three Figs. 9-11, is a copy of the original funding request letter from BNL for the AGS analog ring. This was published with permission of Brookhaven National Laboratory. 
Dr. T.H. Johnson

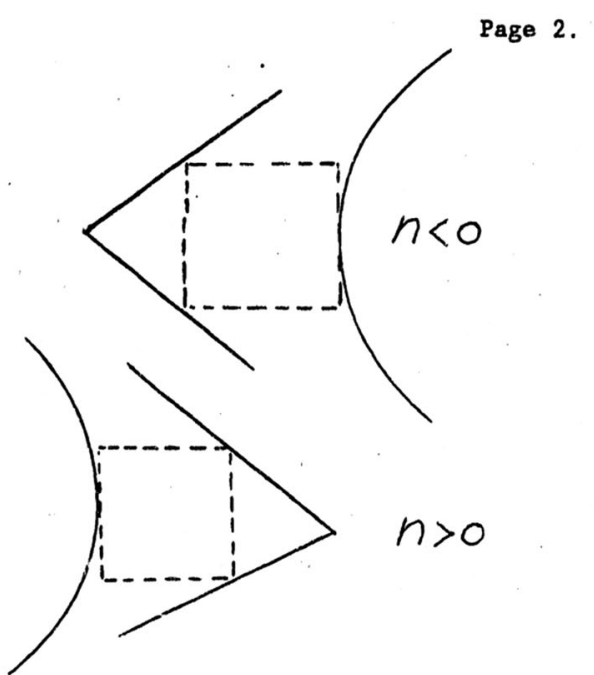

Electrons of about $1 \mathrm{MeV}$ energy would be injected from a small horizontal Van de Graaff generator (of the $2 \mathrm{MeV}$ type manufactured by the High Voltage Engineering Corporation) so that $5 \%$ to $6 \%$ frequency modulation would be required.

Use of a reasonably large radius helps the radio frequency and observing equipment in frequency range where good techniques exist, and permits high n-values which are necessary for strong alternating-gradient focussing.

(This, and phase transition, will not be modeled in the Cornell machine.) A moderate risa rate, consistent with attainable vacuum requirements, still permits the use of small, air-cooled amplifier tubes and a heavily loaded lowQ rf cavity.

A tentative list of parameters is:

Radius of curvature

Over-all diameter

n

No. of periods

No, of straight sections

No. of lenses per period

Length of lens

Length of straight section
$15 \mathrm{ft}$

$45 \mathrm{ft}$

200

37

74

4

$7.6 \mathrm{in}$.

$7.6 \mathrm{in}$

FIG. 9. Haworth letter, page 2. 
Dr. T.H. Johnson

Page 3.

Field strength (magnetic type)

at injection

at $10 \mathrm{MeV}$

10.5 gauss

74 gauss

Field strength (electrostatic type)

at injection

at $10 \mathrm{MeV}$

$3 \mathrm{kV} / \mathrm{cm}$

$22 \mathrm{kV} / \mathrm{cm}$

Rise time

$.01 \mathrm{sec}$

Phase transition energy

$2.8 \mathrm{MeV}$

Frequency (final)

$7 \mathrm{mc}$

Frequency change

5 เ $\%$

Volts/turn

$150 \mathrm{~V}$

$\mathrm{RF}$ power

about $1 \mathrm{kw}$

No. of betatron wavelengths

about 6.2

aperture

$1 \times 1$ in.

Betatron amplitude for $10^{-3} \mathrm{rad}$. error

$0.07 \mathrm{in.}$

Maximum stable amplitude, synchrotron osc. -0.16 in.

Rad:al spacing of betatron resonances about $0.4 \mathrm{in}$.

Vac' um requirement

about $10^{-6} \mathrm{~mm} \mathrm{Hg}$

Total pow ir requirements will be small and available with existing

installations. The test shack seems to be a sultable location since the ring will be erected inside a thin magnetic shield which can be thermally insulated and heated economically.

We estimate the cost to be approximately $\$ 600,000$, distributed as shown in the following table:

Mode1

Staff S. \& W.

Van de Graaff

Other E. \& S.

Shops
Direct Overhead Total

$\begin{array}{rrr}\$ 135,000 & \$ 65,000 & \$ 200,000 \\ 70,000 & - & 70,000 \\ 130,000 & - & 130,000 \\ \underline{135,000} & \$ 65,000 & \frac{200,000}{\$ 470,000} \\ \$ 130,000 & \$ 600,000\end{array}$

FIG. 10. Haworth letter, page 3. 
It is seen that total direct expense would be approximately $\$ 470,000$ and the overhead assigned by our methods of accounting would be approximately $\$ 130,000$. Since the total Laboratory overhead cost would not actually be appreciably affected by the project, the assignment of this overhead would result in relieving Laboratory operations of cost in approximately the same umount. A small but finite savings to operations would also be affected by way of shop charges since approximately one-third of the shop costs represent fixed expenses for foremen, tool crib attendants and other helpers.

The above facts would, of course, reduce the budgetary difficulties in which we find ourselves as a result of the present operational ceilings. Furthermore, the construction of the model would result is some transfers of scientific, technical and shop personnel from the operating program, resulting in further savings. We would naturally like to minimize this by adding new personnel either in operations or for the construction of the model. Allowing for a reasonable compromise in this connection, I belleve that our Physical Research Program could be carried forward without too serious sacrifices by the provision of the $\$ 600,000$ required for the electron model, the additional $\$ 100,000$ promised for Applied Chemistry and $\$ 350,000$ additional operating funds for Applied Physics. Were all of this forthcoming it would not, in my opinion, be necessary to reduce the power level of the reactor but we would endeavor to make all possible economies in equipment and supplies and in overhead costs and would hold physics personnel to present levels except for a few additions in the accelerator development work.

Sincerely yours,

LNH: DS

Leland $\mathrm{J}$, Haworth

cc: E.L. VanHorn

Director

FIG. 11. Haworth letter, page 4. 


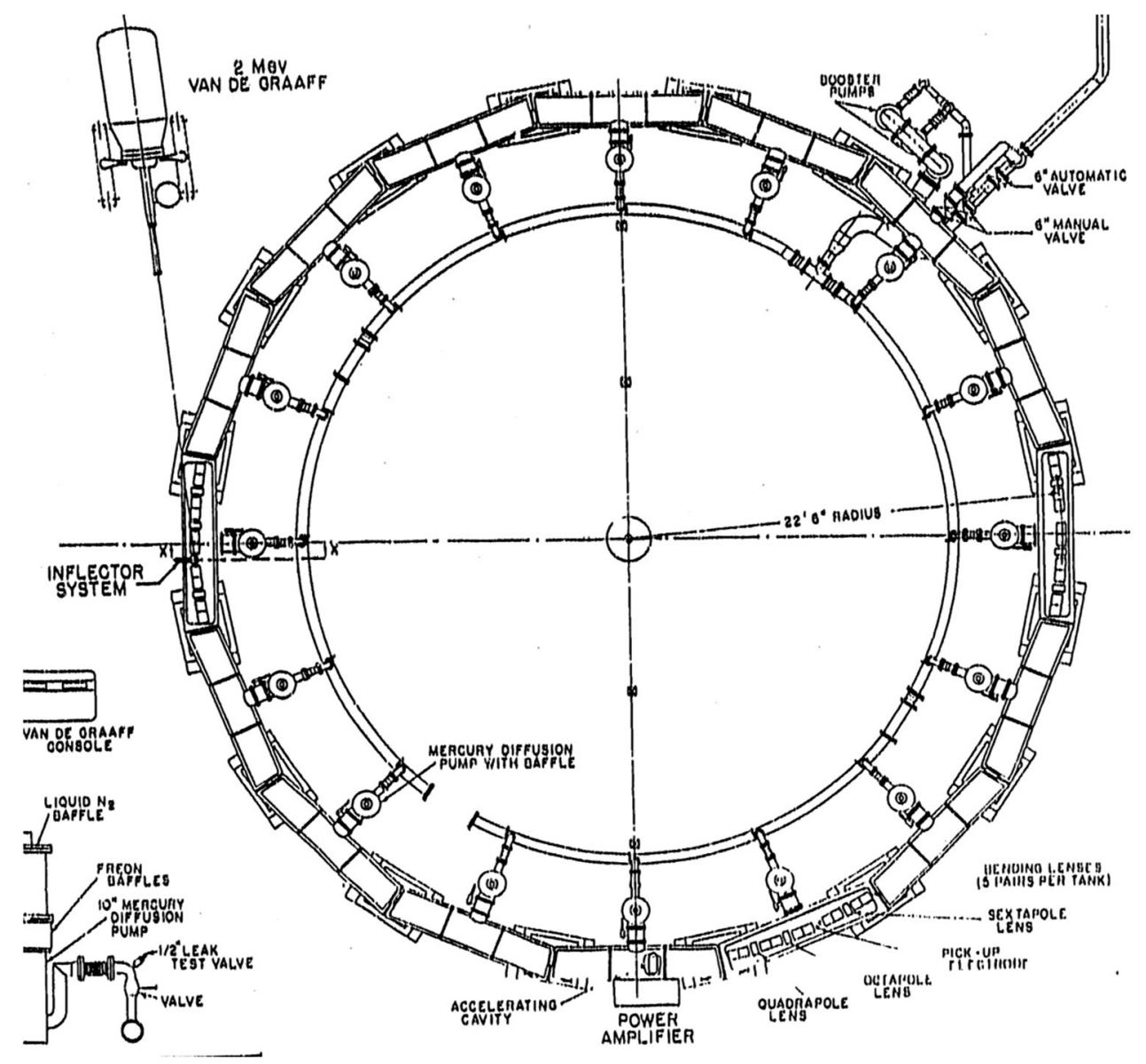

FIG. 12. Electric "lenses" were contained, 10 to a tank, within the 16 vacuum "tanks" making up the full AGS analog ring. Each tank therefore contained $2 \frac{1}{2}$ lattice cells, as well as miscellaneous, special purpose quadrupoles, chromaticity-correcting sextupoles and octupoles. This was published with permission of Brookhaven National Laboratory. 


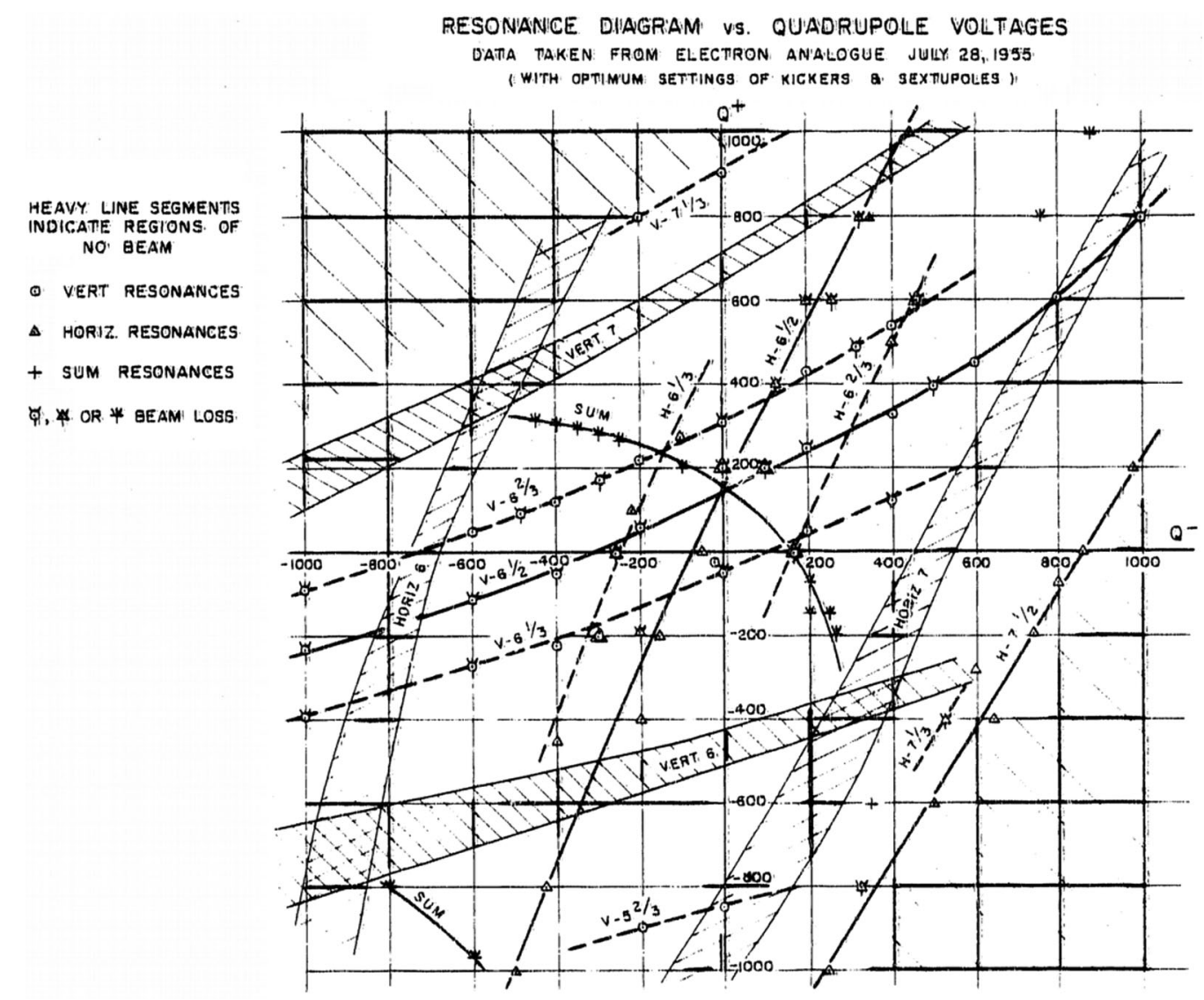

FIG. 13. This figure, from Plotkin [5], with little accompanying explanation, clearly represents the refined analysis of AGS analog machine studies data like that shown in Fig. 2. This was published with permission of Brookhaven National Laboratory.

[1] Storage Ring EDM Collaboration, proposal available from http://www.bnl.gov/edm/files/pdf/proton_EDM_proposal_ 20111027_final.pdf, unpublished (2011).

[2] N. Malitsky and R. Talman, Unified Accelerator Libraries (AIP, Williamsburg, 1996), Vol. 391, https://code.google .com/p/ual/.

[3] W. Morse, All electric magic momentum electron EDM precursor experiment, Brookhaven National Lab Internal Report, 2012.

[4] I. Bazarov, Performance of a high current, low emittance electron gun, Cornell Report, 2013.

[5] M. Plotkin, The Brookhaven electron analogue, Report No. BNL-45058, 1991.

[6] E. Courant, Resonance in the electron analog, BNL Internal Report No. EDC-20, 1955.
[7] W. Morse (private communication).

[8] N. Malitsky, D. Reynolds, and R. Talman, Precise highperformance simulator for EDM experiments, DOE Office of Science Application, 2015.

[9] R. Talman and J. Talman, preceding article, Symplectic orbit and spin tracking code for all-electric storage rings, Phys. Rev. ST Accel. Beams 18, 074003 (2015).

[10] J. Talman and R. Talman, BNL internal reports: UAL/ ETEAPOT results for proton EDM benchmark lattices, 2012, UAL/ETEAPOT proton EDM benchmark comparisons II: Transfer matrices and Twiss functions, 2012; and UAL/ ETEAPOT proton EDM benchmark comparisons III: Dispersion, longitudinal dynamics and synchrotron oscillations, 2012. 